\title{
CLASSIFICATION OF POLARIMETRIC SAR IMAGE BASED ON THE SUBSPACE METHOD
}

\author{
Xu Juan ${ }^{\mathrm{a}, \mathrm{b}, *}$, Li Zhen $^{\mathrm{a}}$, Tian Bangsen ${ }^{\mathrm{a}}$, Chen Quan ${ }^{\mathrm{a}}$, Zhang Ping ${ }^{\mathrm{a}}$ \\ ${ }^{a}$ Institute of Remote Sensing and Digital Earth, Chinese Academy of Sciences, Beijing 100094, China(juanxu, zli, \\ bstian, qchen, pzhang)@ceode.ac.cn \\ ${ }^{\mathrm{b}}$ University of Chinese Academy of Sciences, Beijing, 100049,China
}

KEY WORDS: Polarimetric SAR, Classification, subspace, features

\begin{abstract}
:
Land cover classification is one of the most significant applications in remote sensing. Compared to optical sensing technologies, synthetic aperture radar (SAR) can penetrate through clouds and have all-weather capabilities. Therefore, land cover classification for SAR image is important in remote sensing. The subspace method is a novel method for the SAR data, which reduces data dimensionality by incorporating feature extraction into the classification process. This paper uses the averaged learning subspace method (ALSM) method that can be applied to the fully polarimetric SAR image for classification. The ALSM algorithm integrates three-component decomposition, eigenvalue/eigenvector decomposition and textural features derived from the gray-level cooccurrence matrix (GLCM). The study site, locates in the Dingxing county, in Hebei Province, China. We compare the subspace method with the traditional supervised Wishart classification. By conducting experiments on the fully polarimetric Radarsat-2 image, we conclude the proposed method yield higher classification accuracy. Therefore, the ALSM classification method is a feasible and alternative method for SAR image.
\end{abstract}

\section{INTRODUCTION}

Land cover classification is one of the most significant applications in remote sensing. Remote sensing data obtained from different optical sensors have been commonly used to characterize and quantify land cover information (Saatchi, 1997 and Roberts, 2003). Compared to optical sensing technologies, synthetic aperture radar (SAR) is an effective tool for crop monitoring for its capability to penetrate through clouds and to operate in all-weather conditions. Therefore, land cover classification for SAR images is important in remote sensing.

Various approaches have been used to examine polarimetric SAR data for land cover classification during the past years, such as an entropy based classification (Cloude, 1997), a complex Wishart distribution based classification (Lee, 1994), the support vector machine (SVM) classification (Chen, 2008), subspace pattern recognition method (Watanabe, 1967) and other approaches.

The subspace methods have been used to solve remote sensing image classification problems and have shown potential for efficient classification of high-dimensional remote sensing data (Bagan, 2008). Subspace methods have become popular both for dimensionality reduction and for building classifiers. The basic subspace method is the class-featuring information compression (CLAFIC) (Watanabe, 1967), whose drawback is the subspaces of different classes don't depend on each other. The averaged learning subspace method (ALSM) (Oja, 1983) can overcome this problem and can generate the optimal subspace for each class.

The objective of this paper is to integrate the subspace method, linear polarimetric intensity, polarimetric target decompositions and textural features to improve classification accuracy. A comparison is performed between the subspace method and the traditional supervised Wishart method on the classification of the RadarSat-2 image.

The paper is organized as follows: Section 2 introduces the study site and the data. The methodology is described in Section 3. Then, the experimental results and the conclusion are drawn.

\section{THE STUDY SITE AND DATA SET}

\subsection{The Study Site}

The study site (figure 1) belongs to the North China plain, which is located in Dingxing, Hebei province of northern China $\left(39^{\circ} 01^{\prime}-39^{\circ} 18^{\prime} \mathrm{N}, 115^{\circ} 35^{\prime}-115^{\circ} 54^{\prime} \mathrm{E}\right)$, with a total area of 717 square kilometers. The area is flat and is 13-43 meters above sea level. The climate of this region is temperate semi-arid monsoon. The average annual temperature is $11.7{ }^{\circ} \mathrm{C}$ and the average annual rainfall is about $500 \mathrm{~mm}$, mainly concentrated in the June-August.

\subsection{Experiment Data}

The images of the fine quad-polarimetric RADARSAT-2 (figure 2), acquired on 14 April 2013 (the jointing stage), is utilized in the experiment. The pixel spacing of the image is $4.7 \times 5.1$ meters, and incidence angle is from $44.4^{\circ}-45.7^{\circ}$. Land cover classes in this study area are summarized into four categories: water, peanut, building and farmland. Lots of samples are collected for each category and divide them into two groups, i.e. training and validation respectively. 


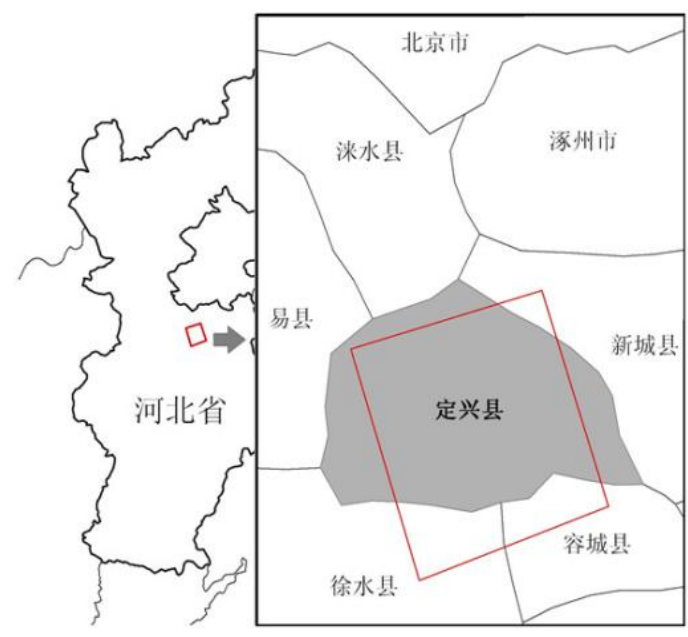

Figure 1. The location of the test site

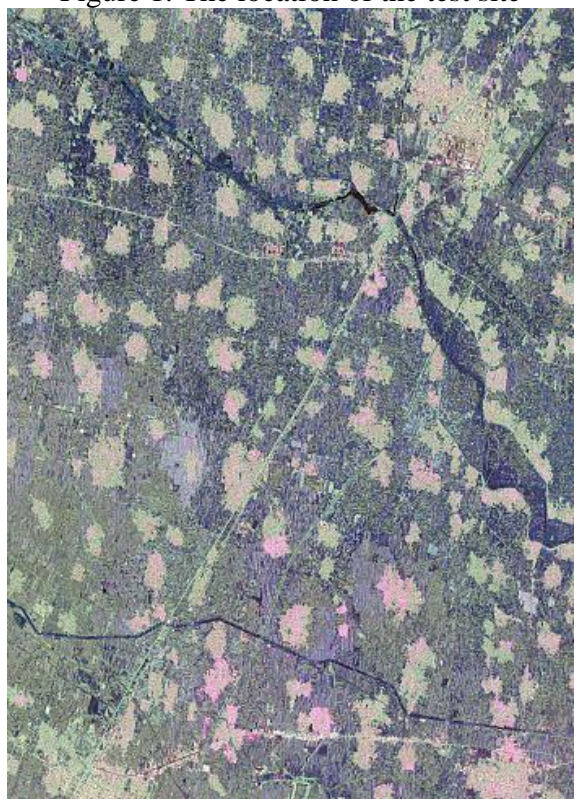

Figure 2. The PauliRGB image of the study site

\section{METHODOLOGY}

\subsection{The subspace method}

The subspace method is a statistical method, it is based on the assumption that the data within each class approximately lie on a lower-dimensional subspace, which tends to be more easily interpretable. It can reduce data dimensionality by incorporating feature extraction into the classification process. Several different subspace methods have been developed. The basic subspace method is the CLAFIC, whose procedure is as follow: A subspace $L_{\mathrm{j}}$ of class $j$ is represented by a matrix $U_{j}$ formed of vectors $u_{1 j}, \ldots, u_{l_{j} j}$. Any vector $x$ of the pattern space $R^{d}$ can then represent as a sum of two vectors, with one belonging to the subspace the other orthogonal to it,

$$
x=\hat{x}_{j}+\tilde{x}_{j}=U_{j} U_{j}^{T} x+\left(I-U_{j} U_{j}^{T}\right) x
$$

The length $\hat{\chi}_{i}$ of can be used as the discriminant function, giving the classification rule for $\mathrm{c}$ classes:

$$
g(x)=\underset{j=1, \ldots, c}{\arg \max } \square U_{j}^{T} x \square^{2}=\underset{j=1, \ldots, c}{\arg \max } \omega_{i j} \sum_{i=1}^{l_{j}}\left(u_{i j}^{T} x\right)^{2}
$$

where the weighting factor $\omega_{i j}=\left(\lambda_{i j} / \lambda_{1 j}\right)^{\rho}$. As the basis vectors $u_{i j}$, the first eigenvectors of the class $j$ correlation matrix $\hat{R}_{j}$ are normally used, corresponding to the largest eigenvalues $\lambda_{1 j}, \ldots, \lambda_{l_{j} j}$ in decreasing magnitude. The correlation matrix can be estimated from the training set of class $j$.

The drawback of the CLAFIC is the subspaces of different classes don't depend on each other. The ALSM, which is an error-driven method, can overcome this problem and can generate the optimal subspace for each class. It changes the subspace by modifying the correlation matrices:

$$
\hat{S}_{j}(k)=\hat{S}_{j}(k-1)+\alpha \sum_{i \in A_{j}} x_{i} x_{i}^{T}-\beta \sum_{i \in B_{j}} x_{i} x_{i}^{T}
$$

where $\alpha$ and $\beta$ are learning parameters. $A_{j}$ indicates vectors which belong to class $j$ but are erroneously classified to some other class, $\beta_{j}$ indicates vectors which are classified to class $j$ but belong to some other class.

\subsection{Features derived from the SAR data}

\subsubsection{H/A/ $\bar{\alpha}$ decomposition}

This decomposition method relies on an eigenvalue analysis of the coherency matrix T. Applying eigenvalue analysis, the mean scattering angel $\bar{\alpha}$, the entropy $H$, and the anisotropy $A$ are defined as:

$$
\begin{gathered}
\bar{\alpha}=\mathrm{P}_{1} \alpha_{1}+\mathrm{P}_{2} \alpha_{2}+\mathrm{P}_{3} \alpha_{3} \\
H=-\sum_{k=1}^{3} P_{k} \log _{3}\left(P_{k}\right) \\
A=\frac{\lambda_{2}-\lambda_{3}}{\lambda_{2}+\lambda_{3}}
\end{gathered}
$$$$
\text { where } \quad P_{K}=\frac{\lambda_{k}}{\sum_{j=1}^{3} \lambda_{j}}
$$ 


\section{$\lambda_{i}(i=1,2,3)$ are the eigenvalues.}

\subsubsection{Freeman-Durden Decomposition}

The Freeman-Durden decomposition models (Freeman, 1998) the covariance matrix as the contribution of three different scattering mechanisms: surface or single-bounce scattering, double-bounce scattering, and volume scattering:

$$
[C]=\left[\begin{array}{ccc}
f_{s}|\beta|+f_{d}|\alpha|^{2}+f_{v} & 0 & f_{s} \beta+f_{d} \alpha+\frac{f_{v}}{3} \\
0 & \frac{2 f_{v}}{3} & 0 \\
f_{s} \beta^{*}+f_{d} \alpha^{*}+\frac{f_{v}}{3} & 0 & f_{s}+f_{d}+f_{v}
\end{array}\right]
$$

The contribution on the dominance in scattering powers of $P_{s}$, $P_{d}$ and $P_{v}$, corresponding to surface, double bounce, and volume scattering can be estimated as follows:

$$
P_{s}=f_{s}\left(1+|\beta|^{2}\right), P_{d}=f_{d}\left(1+|\alpha|^{2}\right), P_{v}=\frac{8}{3} f_{v}
$$

\subsubsection{The GLCM texture}

The GLCM takes into account the specific position of a pixel relative to another. The four features, extracted from the GLCM, are energy, entropy, contrast and homogeneity.

$$
\begin{aligned}
& \text { Energy }=\sum_{i, j=0}^{N-1}\left(P_{i j}\right)^{2} \\
& \text { Entropy }=\sum_{i, j=0}^{N-1}-\ln \left(P_{i j}\right) P_{i j} \\
& \text { Contrast }=\sum_{i, j=0}^{N-1} P_{i j}(i-j)^{2} \\
& \text { Homogeneity }=\sum_{i, j=0}^{N-1} \frac{P_{i j}}{1+(i-j)^{2}}
\end{aligned}
$$

where $P_{i j}$ is element $\mathrm{i}, \mathrm{j}$ of the normalized symmetrical GLCM, $\mathrm{N}$ is the number of gray levels in the image.

\section{EXPERIMENTAL RESULTS}

The main procedure of the proposed classification algorithm is summarized as follow:
1. Filter the image with the J.S. Lee Sigma filtering (Lee, 2009) to reduce speckle noise in the beginning.

2. Deduce the polarimetric features from the RADARSAT-2 SAR images and form a multichannel image.

3. Normalize each pixel to avoid the overflow problem.

4. Generate the subspaces learning and class subspace learning. 5. Analyze and evaluation the result.

A comparison between the proposed method and the Wishart supervised classification is made to evaluate the performance of both methods for land cover classification. The classification map of the proposed method is shown in Fig.2 and the accuracy statistics of these two methods are provided in Table 1 and 2, respectively. Obviously, the overall accuracy of the proposed method is $88.39 \%$, which is higher than that of the Wishart supervised classification, which is $76.70 \%$. The kappa coefficient of the proposed method is 0.85 , whereas that of the Wishart supervised classification is 0.69 .

\section{CONCLUSION}

In this study, we proposed the classification for the fully PolSAR image, which combines ALSM, three-component decomposition, eigenvalue/eigenvector decomposition and textural features. The supervised Wishart classification was also conducted. Experimental results validate the feasibility and accuracy of the proposed method for land cover classification.

\section{ACKNOWLEDGEMENTS}

This research is supported by the National High Technology Research and Development Program ("863 Program," Grant NO. 2011AA120403) and the Chinese Natural Science Foundation Program (Grant NO. 41201447).

Table 2. Classification Accuracy of the Proposed Method

\begin{tabular}{ccccccc}
\hline & \multicolumn{7}{c}{ Reference data } \\
\cline { 2 - 7 } Classified & Water & Buliding & Road & Farmland & Total & UA(\%) \\
\hline Water & 561 & 0 & 0 & 3 & 564 & 99.47 \\
Buliding & 0 & 498 & 68 & 1 & 567 & 87.83 \\
Road & 0 & 40 & 442 & 116 & 598 & 73.91 \\
Farmland & 0 & 9 & 21 & 464 & 494 & 93.93 \\
Total & 561 & 547 & 531 & 584 & 2223 & \\
PA (\%) & 100.00 & 91.04 & 83.24 & 79.45 & & \\
OA(\%) & 88.39 & & & & & \\
Kappa & 0.85 & & & & &
\end{tabular}

Table 3. Classification Accuracy of the Wishart Supervised Classification

\begin{tabular}{ccccccc}
\hline & \multicolumn{6}{c}{ Reference data } \\
\cline { 2 - 7 } Classified data & Water & Buliding & Road & Farmland & Total & UA (\%) \\
\hline Water & 561 & 0 & 0 & 0 & 561 & 100 \\
Buliding & 0 & 531 & 89 & 1 & 621 & 85.51 \\
Road & 0 & 11 & 408 & 378 & 797 & 51.19 \\
Farmland & 0 & 5 & 34 & 205 & 244 & 84.02 \\
Total & 561 & 547 & 531 & 584 & 2223 & \\
PA (\%) & 100.00 & 97.07 & 76.84 & 35.10 & & \\
OA (\%) & 76.70 & & & & & \\
Kappa & 0.69 & & & & &
\end{tabular}




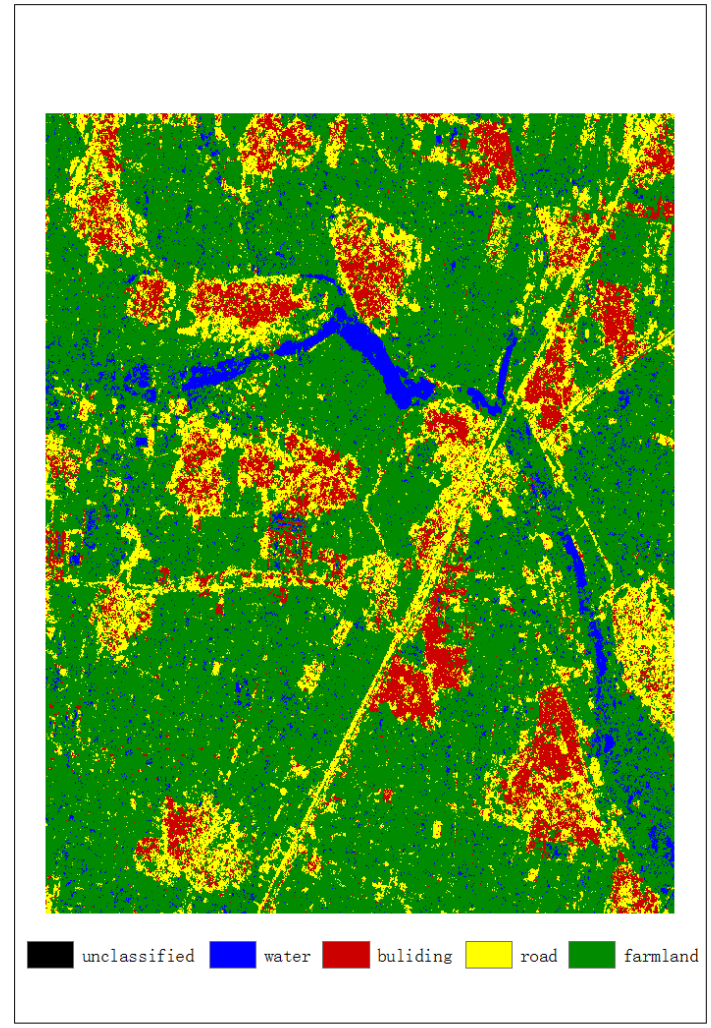

Figure 3. Classification result: (a) proposed method, (b) supervised Wishart classifier

\section{REFERENCES}

Bagan, H., Yasuoka, Y. Endo, T., Wang, X. and Feng, Z., 2008. "Classification of airborne hyperspectral data based on the average learning subspace method," IEEE Geosci. Remote Sens. Lett. , vol. 5, no. 3, pp. 368-372, Jul.

Chen, C. H. and P.-G. P. Ho, 2008. "Statistical pattern recognition in remote sensing," Pattern Recognition, Vol. 41, No. 9, 2731-2741.

Cloude, S. and Pottier, E., 1997. "An entropy based classification scheme for land applications of polarimetric SAR," IEEE Trans. Geosci. Remote Sens. , vol. 35, no. 1, pp. 68-78, Jan.

Freeman, A., and Durden, S., 1998. "A three-component scattering model for polarimetric SAR data", IEEE Trans.Geosci.Remote Sens., vol. 36, no. 3, pp. 963-973.

Lee, J. S., Grunes, M. and Kwok, R., 1994. "Classification of multi-look po-larimetric SAR imagery based on complex Wishart distribution," Int. J. Remote Sens., vol. 15, no. 11, pp. 2299-2311.

Lee, J. S., Wen, J. H. and Ainsworth, T. L., 2009. "Improved sigma filter for speckle filtering of SAR imagery," IEEE Trans. on Geosci. Remote Sens., vol. 47, no. 1, pp. 202-213, Jan.

Oja, E., 1983. Subspace Methods of Pattern Recognition, U.K.: Research Studies Press, Letchworth.
Roberts, D. A., 2003. Keller, M., \& Soares, J. V., "Studies of land-cover, land-use, and biophysical properties of vegetation in the large scale biosphere atmosphere experiment in Amazonia," Remote Sensing of Environment, Vol.87, 377-388.

Saatchi, S. S., Soares, J. V., \& Alves, D. S., 1997. "Mapping deforestation and land use in Amazon rainforest by using SIR-C imagery," Remote Sensing of Environment, Vol. 59, 191-202.

Watanabe, S., Lambert, P. F. and Kulikowski, C. A., 1967. Evaluation and selection of variables in pattern recognition, Computer and Information Sciences II, New York: Academic. 